\title{
Metabolomics perspectives in pediatric research
}

\author{
Sofia Moco' ', Sebastiano Collino', Serge Rezzi' and François-Pierre J. Martin'
}

Increasing evidence points toward the critical and long-term involvement of prenatal and early nutrition and lifestyle on later health and disease risk predisposition. Metabolomics is now a well-established top-down systems biology approach that explores the genetic-environment-health paradigm. The generalization of such approaches has opened new research areas to deepen our current understanding of many physiological processes, as well as foods and nutrient functionalities in target populations. It is envisioned that this will provide new avenues toward preventive medicine and prognostic strategies for tailored therapeutic and personalized nutrition management. The development of systems biology approaches and the new generation of biomarker patterns will provide the opportunity to associate complex metabolic regulations with the etiology of multifactorial pediatric diseases. This may subsequently lead to the development of system mechanistic hypotheses that could be targeted with new nutritional personalized concepts. Therefore, this review aims to describe recent applications of metabolomics in preclinical and clinical fields with insights into disease diagnostics/monitoring and improvement of homeostasis metabolic regulation that may be translatable to novel therapeutic and nutrition advances in pediatric research.

O ver the past decades, awareness about the role of nutrition and lifestyle in health and disease risk management has increased, with key emphasis on the prevention of metabolic disorders, including cardiometabolic diseases and type 2 diabetes (1-3). In parallel, increasing evidence points toward the critical and long-term involvement of early nutrition and lifestyle on later health and disease risk predisposition (4). It therefore becomes pertinent to look at newborn metabolism, development, and nutritional requirements to understand the onset of child and adult physiological conditions. Pregnancy, childbirth, lactation, and human milk composition are influenced by the intake of foods (macro- and micronutrients), which subsequently determine both the short- and long-term health and nutritional outcomes of an individual (5). Therefore, it is essential to build knowledge not only on the metabolic dysregulations but also on the effects of specific foods to comprehensively document the metabolic processes associated with individual health at the different stages of the life cycle (Figure 1). This will allow us to formulate nutritional solutions to improve or circumvent certain metabolic imbalances.
The generalization of systems biology approaches has opened new research areas to further our current understanding of many physiological processes, as well as the functionalities of foods and nutrients. In particular, metabolomics is recognized as a powerful top-down systems biology approach to understand the genetic-environment-health paradigm and to identify clinically relevant biomarkers. Its application in clinical studies is envisioned to shed new light on the regulatory processes of complex mammalian systems with regard to disease etiology, patient stratification, and monitoring. Furthermore, metabolomics offers an unprecedented approach to capture the complex metabolic interactions of the host with its commensal microbial partners, providing a new way to define individual and population phenotypes. This feature is of importance in the early life period for the maturation of the gastrointestinal and immune systems, which are under the influence of the gut microbial ecology. Such data will serve as reference to provide an understanding at the cellular and molecular levels of the relationships between early-life nutritional status and the later disease risk predisposition and to formulate future nutritional concepts. As recently commented by Fanos et al. (6,7), the applications of metabolomics have been so far very promising in the field of neonatology, including intrauterine growth restriction (IUGR), perinatal transition, asphyxia, brain injury and hypothermia, metabolic diseases, and perinatal programming. This review aims to describe the recent applications of metabolomics in preclinical and clinical fields with insights into disease diagnostics/monitoring and improvement of homeostasis by metabolic regulation, which may be translatable in future developments in pediatric research (Table 1).

\section{METABOLOMICS TECHNOLOGIES IN CLINICAL STUDIES}

Metabolomics aims at the quantitative analysis of a large number of low-molecular-weight metabolites participating as substrates or products in metabolic pathways existing in all living systems (8). Specific physiological states, gene expression, and environmental stimuli can cause changes in existing homeostatic conditions, which are ultimately reflected in the metabolic composition of the different biological compartments. Therefore, metabolomics attempts to quantitatively profile small molecules endogenously and exogenously present in a complex biological system; in humans, this relies on the analysis of biological fluids (blood, urine), tissue biopsies, or stools. There are various analytical tools competent in obtaining metabolomics profiles, but 
mass spectrometry and nuclear magnetic resonance $\left({ }^{1} \mathrm{H}-\mathrm{NMR}\right)$ spectroscopy are the most commonly used. Ranging from untargeted to targeted methods, methodologies have been reported for screening the most established biochemical pathways so far: central carbon metabolism, including glycolysis and tricarboxylic acid cycle; amino acid pathways; lipid pathways; and selected secondary metabolism pathways (9-11). Developments in mass spectrometry and NMR metabolomics strategies include increase of sample throughput, sensitivity, resolution, and ability to identify and quantify metabolites from metabolic profiles. Imaging techniques and flux analysis using stable isotopes are parallel technologies to obtain metabolite information (12). Multivariate statistical and bioinformatics techniques are ultimately used for data mining the complex metabolic profiles that encapsulate information on genetics, environmental factors, gut microbiota activity, lifestyle, and food habits. This combined strategy sustains

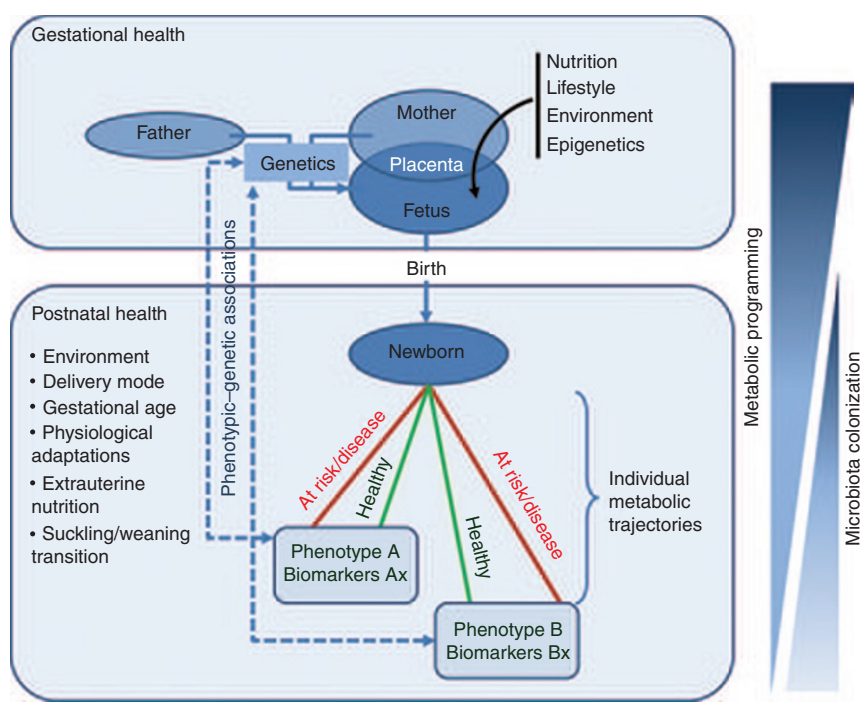

Figure 1. Biomarker strategy for future stratified neonatology and pediatrics health care. Metabolic biomarkers should capture relevant biological events related to developmental biology during both gestational and postnatal life. These markers describe phenotypes based on comprehensive modeling of physiological adaptations of the host, metabolic programming events, and gut microbiota colonization under a dynamic trend (e.g., metabolic trajectories). Once validated, indicative biomarkers will define stratified phenotypic groups related to genetic and metagenomic backgrounds. These stratified phenotypes are the basis of tailor-made health and nutritional care. the complex process of identifying emerging biomarkers indicative of the individual response to specific physiological factors and/or nutritional interventions, and for the elaboration of biological outcomes. In the clinical setting, mass spectrometry- and NMR-based metabolomics have been used in studying a variety of challenges that affect human health, such as diet, metabolic syndrome, cancer, aging, and growth and development (1).

The application of metabolomics analysis in pediatrics is suitable due to the simplicity, safety, and noninvasiveness of the approach, using, for instance, sterile cotton wool balls in the infants' diapers (6). According to the type of sample collection (spot or 24-h urine), urinary metabolic profiles give an approximate time-averaged representation of the recent homeostatic metabolic changes of the individual, in addition to carrying indirect information on the gut microbial metabolic activities, as observed through the excretion of many polar microbial-mammalian cometabolites. The application of metabolomics for blood plasma analysis is more invasive but remains largely applicable in the pediatric fields, mainly through technological development requiring low sample volumes. For instance, analyses can be conducted on blood serum, plasma, or even blood spots (collected using Gutri paper or capillaries in combination with finger prick). Analysis of stool samples is also widely generalized and provides information on digestive and gut microbial metabolism, but often it is difficult to procure cooperation regarding the provision of samples from children and adults. Finally, metabolomics analysis is also applicable to food itself to identify major nutritional constituents and/or particular metabolites showing significant bioactivity, including milk, cereals, wine, fruits, vegetables, and meat (13). This is of particular relevance for the analysis of breast milk (14) in order to explore the relationship among mother's nutrition, breastfeeding, and child's nutrition for optimal growth and development. Human milk varies in composition, time, and volume, and is the first source of nutrient for the newborn. Control of the sampling time and the aliquots should be aimed for in order to obtain representative samples for metabolomics analysis.

\section{EARLY NUTRITION CASCADES INTO LONG-TERM HEALTH EFFECTS}

Nutrition during early development not only has shortterm effects on growth, body composition, and maturation

Table 1. Overview of relevant research areas in pediatrics covered by metabolomics

\begin{tabular}{ll}
\hline Research area & Relevant matrices for metabolomics analysis \\
\hline Metabolic programming & $\begin{array}{l}\text { Mother: plasma, serum, amniotic fluid; infant: urine, plasma, } \\
\text { serum, cord blood }\end{array}$ \\
$\begin{array}{ll}\text { Perinatal environment and monitoring disorders for low } \\
\text { birth weight babies, intrauterine growth retardation }\end{array}$ & $\begin{array}{l}\text { Mother: plasma, serum, amniotic fluid; infant: urine, plasma, } \\
\text { serum, cord blood }\end{array}$ \\
$\begin{array}{l}\text { Perinatal environment and monitoring disorders for } \\
\text { overweight and obesity risk management }\end{array}$ & $\begin{array}{l}\text { Mother: plasma, serum, amniotic fluid; infant: urine, plasma, } \\
\text { serum, cord blood }\end{array}$ \\
$\begin{array}{l}\text { Gestational age: preterm/full-term } \\
\text { Delivery mode: vaginal/cesarean }\end{array}$ & Mother: breast milk; infant: urine, plasma, serum, stool \\
Feeding: breast-fed/formula-fed & Mother: breast milk; infant: urine, plasma, serum, stool \\
Inborn errors of metabolism & Mother: breast milk; infant: urine, plasma, serum, stool \\
Gut colonization & Infant: urine \\
\hline
\end{tabular}


of biological functions but also exerts long-term effects on health, disease, and mortality risks in adulthood, a phenomenon called "metabolic programming" (5). The interaction of nutrients, gene expression, and metabolism may form the basis of many of these programming effects, which ultimately determine the physiological status of an individual.

In clinical studies, metabolomics was recently employed to demonstrate how maternal blood composition during pregnancy might provide novel insights for predicting and/or elucidating the impact of maternal health and nutritional status on childbirth, growth, and development. For instance, the mother's blood composition during the third trimester of pregnancy showed that circulating levels of lipids (glycerophospholipids, sphingolipids, sterols), hormones (progesterone), and vitamins (vitamin D) were related to the rate of poor pregnancy outcomes (15). Similarly, blood plasma of mothers during their first trimester was associated with the risk of early onset of preeclampsia (16-18). Using different methodologies, the concentrations of specific carnitines, amino acids (alanine, phenylalanine glutamate, or methionine), and energy intermediates (citrate, glycerol, and hydroxyisovalerate) were highly predictive of preeclampsia. Metabolomics analysis of maternal urine and plasma was also conducted during the second trimester of pregnancy to identify markers of fetal malformations (19). Increased excretion of hypoxanthine, stimulated gluconeogenesis (ketogenesis, tricarboxylic acid cycle, glucogenic amino acids), and altered blood lipid composition (energy and cell membrane composition) were identified as imprinted signatures of fetal disorders in maternal biofluids.

The biological processes involved in the incidence of low birth weight babies and IUGR are being further scrutinized due to the high rates of morbidity and mortality, low efficiency of food utilization, and the long-term health consequences for the offspring. For instance, epidemiological studies on twins have shown that the twin with the lower birth weight is more susceptible to developing type 2 diabetes compared to the one with higher birth weight (20). Analysis of umbilical cord blood soon after birth allowed the exploration of differences in the motherfetus interaction that may be involved in this condition (21). The authors showed that the circulating levels of amino acids, including phenylalanine, tryptophan, and methionine, were strikingly different between IUGR and appropriate for gestational age fetuses. Studies in pig models described the potential use of umbilical vein blood metabolomics analysis for designing effective strategies to prevent, diagnose, and treat IUGR in mammalian species (22). In addition to the lack of knowledge of the multifactorial events involved in IUGR, there is also a lack of understanding of the needs of IUGR infants (23). Recently, urine metabolomics provided novel insights into metabolic abnormalities experienced by IUGR infants and on which further nutritional adjustment could be conceptualized. In particular, urinary excretion of myo-inositol, sarcosine, creatine, and creatinine were highlighted, which may refer putatively to signaling functions, betaine/homocysteine metabolism, and muscle/free fat mass metabolism, respectively. Such a concept can be exemplified through the analysis of the metabolic response of offspring to maternal protein restriction during pregnancy and lactation in the IUGR rat model (24). Offspring who suffered from maternal protein deprivation during pregnancy were born with IUGR and showed a rapid catch-up growth. Their metabolome showed minimal changes early in life but more pronounced changes during adulthood. Animals who experienced maternal protein deprivation during pregnancy and lactation showed IUGR with slow postnatal growth and marked metabolome differences during suckling, but none at birth. Therefore, such investigations describe how long-term regulation in feeding behavior and lipid metabolism in IUGR depends on postnatal velocity. Horgan et al. (25) further exemplified how blood metabolomics could be relevant for the study of smallfor-gestational-age babies and the related risks of perinatal morbidity and mortality, cardiovascular complications, and diabetes in later life. The authors described how blood plasma composition in early pregnancy preceding the onset of being small for gestational age could unravel novel features of pathogenesis relevant for a future presymptomatic screening test (25). Maybe not surprisingly, the blood analysis pointed toward the class of compounds that was previously commented upon, including sphingolipids, phospholipids, carnitines, and fatty acids, which are nowadays highlighted in many cardiometabolic disorders and/or insulin resistance biological processes.

During the early neonatal period, infants born premature are at risk of infection, lung disease, gastrointestinal disease, neurological bleeding, and cardiovascular compromise. Among these complications, necrotizing enterocolitis, the most common gastrointestinal emergency in the newborn infant, is a disorder manifested by ischemic necrosis of the intestinal mucosa. Ioannou et al. (26) measured plasma citrulline levels in 17 preterm neonates with necrotizing enterocolitis stage II during the entire course of the disease. Neonates experiencing necrotizing enterocolitis presented significantly lower mean citrulline levels as compared with controls, prompting identification of this molecule as a potential metabolic signature of necrotizing enterocolitis. Metabolomics in pediatric research is also finding new avenues in managing complications of very low birth weight. Among the many comorbidities, respiratory distress syndrome and its main complication, bronchopulmonary dysplasia, represent one of the main causes of neonatal mortality and morbidity. The potential of metabolomics as a tool in the management of sick premature infants was discussed by Fabiano et al. (27). The authors analyzed bronchoalveolar lavage fluid samples collected at birth before surfactant administration, postsurfactant during mechanical ventilation, and at extubation time points. Although a specific fingerprint of bronchoalveolar lavage fluid was found, a metabolic signature was also found similar to that of children with cystic fibrosis (28), suggesting similarities in the inflammation processes between the two studied populations. Recently, Atzori et al. (29) applied an NMR metabolomics approach to characterize the metabolite patterns associated with different nephrouropathies. As compared with healthy children, children with disease displayed differences in purine and pyridine metabolism and variations of the urea cycle, suggesting alterations in the development and growth of these children. 
In clinical practice, many studies have also focused on investigating amniotic fluid features, with metabolomics used for identifying markers of fetal malformations, prediagnostic gestational diabetes, and preterm delivery (30). Metabolic profiles of amniotic fluids collected during the second trimester of pregnancy were imprinted according to fetal malformations and gestational diabetes, per different energy, kidney, amino acid, and nucleotide metabolism. Similarly, profiles of amniotic fluid from women with spontaneous preterm labor with intact membranes demonstrated again their relevance to assessing the risk of preterm delivery in the presence or absence of infection and inflammatory conditions (31).

In addition to IUGR, fetal macrosomia, or being large for gestational age, confers the risk of childhood obesity. A metabolomics study on the urine of women on the first trimester could predict the later development of fetal macrosomia, leading to the hypothesis that when diagnosed on time, possible nutritional intervention can be administered to attenuate the development of such condition (32). The prevalence of both child and adult obesity is rising worldwide, with an estimated 155 million children being overweight and 30-45 million being classified as obese $(33,34)$. Childhood overweight is a strong predictor of adult obesity (33) and its level will continue to rise, with severe health consequences, unless urgent action is taken. Following the obesity epidemic trend, overweight and obesity are becoming common in pregnant women $(35,36)$, with maternal obesity in early pregnancy being associated with a four- and twofold increased risk of childhood overweight (37) and obesity (38), respectively, likely through genetic as well as pre- and postnatal environment factors. Of note, overweight/obese women with normal glucose tolerance levels have neonates who are heavier than lean/average weight women because of increased adiposity (39). Breastfeeding seems to reduce the magnitude of risk of being overweight during childhood in children from obese mothers (37). Nevertheless, obese women have difficulties in initiating and maintaining breastfeeding, likely because of defects in hormonal response to suckling, birth complications, and psychological factors $(40,41)$. Noninvasive application of metabolomics to urine and stool profiling will enable the monitoring of the metabolic response and requirements of infants during growth and subsequently lead to novel mechanistic hypotheses that could be targeted with new nutritional concepts.

\section{DIET AND GUT COLONIZATION IMPACT GROWTH AND DEVELOPMENT}

In addition to the course of pregnancy and childbirth, metabolomics approaches may provide novel insights into postnatal events such as lactation, exposure to human milk, exposure to macro- and micronutrients, and gut colonization leading to short- and long-term health outcomes. Birth represents the transition between a sterile fetal environment and a microbeand substrate-rich environment. It is at birth that the colonization, development, and maturation of the newborn's gastrointestinal tract begins and the gut undergoes extensive modulation, continuing throughout the first 2 y of life. An adult's gut can account for $\sim 100$ trillion cells, mostly bacteria (42). Recent associations have implicated the gut microbiota in a wealth of human conditions and diseases (43). Therefore, it becomes important to understand the contribution of the gut colonization to the overall growth and development of the infant. Starting immediately upon delivery, the infant's gut progressively passes from an aerobic to an anaerobic environment, as the colonization intensifies. Various factors that affect gut colonization, such as gestational age, mode of delivery, feeding regimen, maternal diet/weight, probiotic and prebiotic use, antibiotic exposure, and environment in general (44), might modulate gut microbial composition into a healthy or at-risk status.

Preterm infants acquire a rather diverse bacterial community after birth, as a result of hospitalization, but tend to develop a similar microbiome to that of full-term infants over time. The interindividual bacterial composition in hospitalized preterm infants is more similar in comparison with breast-fed, full-term infants and is not necessarily influenced by birth weight, diet, or antibiotic treatment (45). Likewise, a ${ }^{1} \mathrm{H}-\mathrm{NMR}$ metabolomics study comparing preterm and term urine of infants led to differences in metabolites known for their association with gut microbial activity $(46,47)$.

In vaginal delivery, as the babies are exposed to vaginal and fecal microbes from the mother, a strong maternal signature is found, as compared with cesarean-born babies. Vaginally delivered infants acquired bacterial communities resembling their own mother's vaginal microbiota. Cesarean-born infants harbored bacterial communities similar to those found on the skin surface (48), lower numbers of beneficial bacteria, and a high proportion of potential pathogens (49). These microbial differences may account for increased incidence of food allergy, asthma, and type 1 diabetes (50). A close relationship between the mother's and child's body composition was found. Children of overweight mothers tend to be overweight or heavier at birth and with marked differences in the gut microbiota, with inferred effects on gut functional ecology and predisposition to metabolic disorders (51).

The feeding choice also has an impact on the infant's gut microbiota, in which differences between breastfeeding and formula feeding can be identified. Breastfeeding is described as the gold standard of infant nutrition, as recommended by the World Health Organization. Differences in mother's milk composition as compared to that of formula lead to preferential predominance of certain gut microbes. Bifidobacteria, human milk oligosaccharide specialists, are more abundant in breast-fed infants, whereas formula-fed infants have a higher prevalence of Escherichia coli and Clostridia species such as Clostridium difficile (49). Although the addition of probiotics and prebiotics during early feeding may beneficially modulate the intestinal microbiota composition in infants by reducing the numbers of potential pathogens, antibiotics affect the gut microbial ecology in a profound and adverse way. Tanaka et al. (52) conducted a study in newborns in which a subset was administered a broad-spectrum antibiotic for the first $4 \mathrm{~d}$ of life. Even after a month, antibiotic-treated subjects denoted a distinct microbiota population given the high presence of 
Enterobacteriaceae as compared with the control groups. These findings suggest that antibiotics may provoke long-lasting and possibly adverse effects in gut microbial composition of newborns.

The first $2 \mathrm{y}$ of life represent a window of opportunity during which the gut microbial composition can be positively modulated through diet and lifestyle. Nevertheless, the concept of a "healthy microbiome" for an infant remains to be better understood. The importance of the evolution of the gut ecology to the immune system, growth, and development of the infant remains to be unraveled. Temporal studies, looking at the metabolism of the mother and child, including breast milk, could aid in accessing a wider view on gut colonization and its impact on the infant's health. The gut could then be modulated by providing food supplements, leading to preferential growth of specific beneficial gut bacteria.

\section{METABOLOMICS APPLICATION IN NEONATAL SCREENING AND PEDIATRIC MEDICINE}

Metabolomics has also found powerful applications in detecting inborn errors of metabolism, where diagnosis is based on monitoring abnormal concentrations of metabolites in biofluids. The diseases are caused by deficient enzyme activity in intermediate metabolism pathways, affecting about 1 in every 5,000 babies born (53). These defects can cause an increase in accumulation of toxic compounds, resulting in serious and, in many cases, fatal diseases, of early life. Diagnosis of these compounds in early stages is therefore of crucial importance. One of the first studies to apply NMR spectroscopy as a screening method for inborn errors was conducted by Constantinou et al. (54) to discriminate blood samples obtained from patients with phenylketonuria and maple syrup urine disease; greater concentrations of branched amino acids were observed in patients with maple syrup urine disease. Recently, Schulze et al. (55) determined the impact of expanded newborn screening on the overall detection rate of inborn errors of metabolism in a German population. Data on 250,000 newborns who underwent expanded screening for 23 inborn errors of metabolism, including amino acidemias, fatty acid oxidation disorders, and organic acidurias, were reported over a 42-mo period. Inborn errors of metabolism were confirmed in 106 newborns and 61 of them could have benefited from the screening if treated on time. Sholmi et al. (56) developed a novel computational method that is capable of predicting 176 possible dysfunctional enzymes related to the onset of inborn errors of metabolism by measuring the concentration of 233 metabolites.

Recently, autism was studied using metabolomics tools. Autism typically develops during the first 3 y of life and is characterized by a myriad of deficits in language/communication skills, social detachment, and repetitive and stereotypic behaviors. The etiopathology of autism is multifactorial and has been linked to genetic abnormalities and inborn errors of metabolism (57). Investigations in individuals diagnosed with autism together with their non-autistic siblings demonstrated a profound alteration in the tryptophan-nicotinic acid and sulfur metabolic pathways (58). Most importantly, metabolic phenotype differences were observed between autistic and control children, with perturbations in the relative patterns of urinary metabolites related to the gut microbiota, providing novel insights into the role of the gut-brain axis in the etiology of several diseases.

PERSPECTIVES: NEED FOR NEW PREDICTIVE, MONITORING, AND MECHANISTIC BIOMARKERS

Metabolomics is increasingly becoming popular in neonatology due to its unique ability to generate functional and system readouts of newborns, building the elementary steps for future personalized prenatal, neonatal, and pediatric care. Yet the clinical translation of this unprecedented source of metabolic knowledge, which may be used in the near future in clinical practices for neonatal and pediatric health care, requires proper addressing of the dynamics of physiological and biological adaptations from fetal to postnatal life, including perinatal programming. This is a key period in defining individual metabolic set points that will determine the likelihood of developing a particular disease later in life. In this, the lessons learned and to be learned from epidemiological metabolomic studies, including those on twin cohorts, will help in delineating the interaction of nutrients, gene expression, and metabolism from environmental pressures that ultimately determine the physiological and the disease risk status of neonates and children. Furthermore, metabolic phenotypes will need to capture these dynamics at the molecular and system levels, requiring longitudinal sampling and proper assessment of inherent interindividual variability. Therefore, advances in linking metabolite data to known and validated clinically relevant indexes will have to be seriously considered in addition to consolidating metabolomics and metabolic markers in clinical translation/applications.

In conclusion, the development of systems biology approaches and the new generation of biomarker patterns will provide the opportunity to associate complex metabolic regulations with the etiology of multifactorial pediatric diseases. This will subsequently lead to the development of system mechanistic hypotheses that could be targeted with new nutritional personalized concepts.

Disclosure: All the authors work for Nestlé Institute of Health Sciences S.A.

\section{REFERENCES}

1. Collino S, Martin FP, Rezzi S. Clinical Metabolomics paves the way towards future healthcare strategies. Br J Clin Pharmacol 2012; e-pub ahead of print 20 February 2012.

2. Martin FP, Collino S, Rezzi S, Kochhar S. Metabolomic applications to decipher gut microbial metabolic influence in health and disease. Front Physiol 2012;3:113.

3. Holmes E, Wilson ID, Nicholson JK. Metabolic phenotyping in health and disease. Cell 2008;134:714-7.

4. Koletzko B, Brands B, Poston L, Godfrey K, Demmelmair H; Early Nutrition Project. Early nutrition programming of long-term health. Proc Nutr Soc 2012;71:371-8.

5. Koletzko B, Aggett PJ, Bindels JG, et al. Growth, development and differentiation: a functional food science approach. Br J Nutr 1998;80:Suppl 1:S5-45.

6. Fanos V, Barberini L, Antonucci R, Atzori L. Metabolomics in neonatology and pediatrics. Clin Biochem 2011;44:452-4. 
7. Fanos V, Antonucci R, Barberini L, Noto A, Atzori L. Clinical application of metabolomics in neonatology. J Matern Fetal Neonatal Med 2012;25:Suppl 1:104-9.

8. Nicholson JK, Lindon JC, Holmes E. 'Metabonomics': understanding the metabolic responses of living systems to pathophysiological stimuli via multivariate statistical analysis of biological NMR spectroscopic data. Xenobiotica 1999;29:1181-9.

9. Buescher JM, Moco S, Sauer U, Zamboni N. Ultrahigh performance liquid chromatography-tandem mass spectrometry method for fast and robust quantification of anionic and aromatic metabolites. Anal Chem 2010;82:4403-12.

10. Wenk MR. The emerging field of lipidomics. Nat Rev Drug Discov 2005;4:594-610.

11. Moco S, Bino RJ, Vorst $\mathrm{O}$, et al. A liquid chromatography-mass spectrometry-based metabolome database for tomato. Plant Physiol 2006;141:1205-18.

12. Moco S, Bino RJ, De Vos RCH, Vervoort J. Metabolomics technologies and metabolite identification. Trends Analytic Chem 2007;26:855-66.

13. Herrero M, Simó C, García-Cañas V, Ibáñez E, Cifuentes A. Foodomics: MS-based strategies in modern food science and nutrition. Mass Spectrom Rev 2012;31:49-69.

14. Marincola FC, Noto A, Caboni P, et al. A metabolomic study of preterm human and formula milk by high resolution NMR and GC/MS analysis: preliminary results. J Matern Fetal Neonatal Med 2012;25:Suppl 5:62-7.

15. Heazell AE, Bernatavicius G, Warrander L, Brown MC, Dunn WB. A metabolomic approach identifies differences in maternal serum in third trimester pregnancies that end in poor perinatal outcome. Reprod Sci 2012;19:863-75.

16. Bahado-Singh RO, Akolekar R, Mandal R, et al. Metabolomics and firsttrimester prediction of early-onset preeclampsia. J Matern Fetal Neonatal Med 2012;25:1840-7.

17. Odibo AO, Goetzinger KR, Odibo L, et al. First-trimester prediction of preeclampsia using metabolomic biomarkers: a discovery phase study. Prenat Diagn 2011;31:990-4.

18. Kenny LC, Broadhurst DI, Dunn W, et al.; Screening for Pregnancy Endpoints Consortium. Robust early pregnancy prediction of later preeclampsia using metabolomic biomarkers. Hypertension 2010;56: 741-9.

19. Diaz SO, Pinto J, Graça G, et al. Metabolic biomarkers of prenatal disorders: an exploratory NMR metabonomics study of second trimester maternal urine and blood plasma. J Proteome Res 2011;10:3732-42.

20. Bo S, Cavallo-Perin P, Scaglione L, Ciccone G, Pagano G. Low birthweight and metabolic abnormalities in twins with increased susceptibility to Type 2 diabetes mellitus. Diabet Med 2000;17:365-70.

21. Favretto D, Cosmi E, Ragazzi E, et al. Cord blood metabolomic profiling in intrauterine growth restriction. Anal Bioanal Chem 2012;402:1109-21.

22. Lin G, Liu C, Feng C, et al. Metabolomic analysis reveals differences in umbilical vein plasma metabolites between normal and growth-restricted fetal pigs during late gestation. J Nutr 2012;142:990-8.

23. Dessì A, Atzori L, Noto A, et al. Metabolomics in newborns with intrauterine growth retardation (IUGR): urine reveals markers of metabolic syndrome. J Matern Fetal Neonatal Med 2011;24:Suppl 2:35-9.

24. Alexandre-Gouabau MC, Courant F, Le Gall G, et al. Offspring metabolomic response to maternal protein restriction in a rat model of intrauterine growth restriction (IUGR). J Proteome Res 2011;10:3292-302.

25. Horgan RP, Broadhurst DI, Walsh SK, et al. Metabolic profiling uncovers a phenotypic signature of small for gestational age in early pregnancy. J Proteome Res 2011;10:3660-73.

26. Ioannou HP, Diamanti E, Piretzi K, Drossou-Agakidou V, AugoustidesSavvopoulou P. Plasma citrulline levels in preterm neonates with necrotizing enterocolitis. Early Hum Dev 2012;88:563-6.

27. Fabiano A, Gazzolo D, Zimmermann LJ, et al. Metabolomic analysis of bronchoalveolar lavage fluid in preterm infants complicated by respiratory distress syndrome: preliminary results. J Matern Fetal Neonatal Med 2011;24:Suppl 2:55-8.

28. Wolak JE, Esther CR Jr, O’Connell TM. Metabolomic analysis of bronchoalveolar lavage fluid from cystic fibrosis patients. Biomarkers 2009;14:55-60.
29. Atzori L, Antonucci R, Barberini L, et al. 1H NMR-based metabolic profiling of urine from children with nephrouropathies. Front Biosci (Elite Ed) 2010;2:725-32.

30. Graca G, Duarte IF, Barros AS, et al. Impact of prenatal disorders on the metabolic profile of second trimester amniotic fluid: a nuclear magnetic resonance metabonomic study. J Proteome Res 2010;9:6016-24.

31. Romero R, Mazaki-Tovi S, Vaisbuch E, et al. Metabolomics in premature labor: a novel approach to identify patients at risk for preterm delivery. J Matern Fetal Neonatal Med 2010;23:1344-59.

32. Walsh JM, Brennan L, Mahony RM, Foley ME, McAuliffe FM. First trimester maternal urinary metabolomic profile to predict macrosomia. Arch Dis Child Fetal Neonatal Ed 2011;96:Fa127.

33. Whitaker RC, Wright JA, Pepe MS, Seidel KD, Dietz WH. Predicting obesity in young adulthood from childhood and parental obesity. N Engl J Med 1997;337:869-73.

34. Lobstein T, Baur L, Uauy R; IASO International Obesity TaskForce. Obesity in children and young people: a crisis in public health. Obes Rev 2004;5:Suppl 1:4-104.

35. Callaway LK, Prins JB, Chang AM, McIntyre HD. The prevalence and impact of overweight and obesity in an Australian obstetric population. Med J Aust 2006;184:56-9.

36. Sebire NJ, Jolly M, Harris JP, et al. Maternal obesity and pregnancy outcome: a study of 287,213 pregnancies in London. Int J Obes Relat Metab Disord 2001;25:1175-82.

37. Li C, Kaur H, Choi WS, Huang TT, Lee RE, Ahluwalia JS. Additive interactions of maternal prepregnancy BMI and breast-feeding on childhood overweight. Obes Res 2005;13:362-71.

38. Whitaker RC. Predicting preschooler obesity at birth: the role of maternal obesity in early pregnancy. Pediatrics 2004;114:e29-36.

39. Sewell MF, Huston-Presley L, Super DM, Catalano P. Increased neonatal fat mass, not lean body mass, is associated with maternal obesity. Am J Obstet Gynecol 2006;195:1100-3.

40. Rasmussen KM, Kjolhede CL. Prepregnant overweight and obesity diminish the prolactin response to suckling in the first week postpartum. Pediatrics 2004;113:e465-71.

41. Rasmussen KM, Hilson JA, Kjolhede CL. Obesity as a risk factor for failure to initiate and sustain lactation. Adv Exp Med Biol 2002;503: 217-22.

42. Tannock GW. New perceptions of the gut microbiota: implications for future research. Gastroenterol Clin North Am 2005;34:361-82, vii.

43. Holmes E, Kinross J, Gibson GR, et al. Therapeutic modulation of microbiota-host metabolic interactions. Sci Transl Med 2012;4:137rv6.

44. Fouhy F, Ross RP, Fitzgerald GF, Stanton C, Cotter PD. Composition of the early intestinal microbiota: knowledge, knowledge gaps and the use of high-throughput sequencing to address these gaps. Gut Microbes 2012;3:203-20.

45. Schwiertz A, Gruhl B, Löbnitz M, Michel P, Radke M, Blaut M. Development of the intestinal bacterial composition in hospitalized preterm infants in comparison with breast-fed, full-term infants. Pediatr Res 2003;54:393-9.

46. Atzori L, Antonucci R, Barberini L, et al. 1H NMR-based metabolomic analysis of urine from preterm and term neonates. Front Biosci (Elite Ed) 2011;3:1005-12.

47. Nicholson JK, Holmes E, Kinross J, et al. Host-gut microbiota metabolic interactions. Science 2012;336:1262-7.

48. Dominguez-Bello MG, Costello EK, Contreras M, et al. Delivery mode shapes the acquisition and structure of the initial microbiota across multiple body habitats in newborns. Proc Natl Acad Sci USA 2010;107:11971-5.

49. Penders J, Thijs C, Vink C, et al. Factors influencing the composition of the intestinal microbiota in early infancy. Pediatrics 2006;118:511-21.

50. Boutsikou T, Malamitsi-Puchner A. Caesarean section: impact on mother and child. Acta Paediatr 2011;100:1518-22.

51. Collado MC, Isolauri E, Laitinen K, Salminen S. Effect of mother's weight on infant's microbiota acquisition, composition, and activity during early infancy: a prospective follow-up study initiated in early pregnancy. Am J Clin Nutr 2010;92:1023-30. 
52. Tanaka S, Kobayashi T, Songjinda P, et al. Influence of antibiotic exposure in the early postnatal period on the development of intestinal microbiota. FEMS Immunol Med Microbiol 2009;56:80-7.

53. Clague A, Thomas A. Neonatal biochemical screening for disease. Clin Chim Acta 2002;315:99-110.

54. Constantinou MA, Papakonstantinou E, Benaki D, et al. Application of nuclear magnetic resonance spectroscopy combined with principal component analysis in detecting inborn errors of metabolism using blood spots: a metabonomic approach. Anal Chim Acta 2004;511:303-12.

55. Schulze A, Lindner M, Kohlmüller D, Olgemöller K, Mayatepek E, Hoffmann GF. Expanded newborn screening for inborn errors of metabolism by electrospray ionization-tandem mass spectrometry: results, outcome, and implications. Pediatrics 2003;111(6 Pt 1): 1399-406.

56. Shlomi T, Cabili MN, Ruppin E. Predicting metabolic biomarkers of human inborn errors of metabolism. Mol Syst Biol 2009; 5:263.

57. Kidd PM. Autism, an extreme challenge to integrative medicine. Part: 1 : The knowledge base. Altern Med Rev 2002;7:292-316.

58. Oresic M, Simell S, Sysi-Aho M, et al. Dysregulation of lipid and amino acid metabolism precedes islet autoimmunity in children who later progress to type 1 diabetes. J Exp Med 2008;205:2975-84. 